\title{
Controller Complexity Reduction for Piecewise Affine Systems Through Safe Region Elimination
}

\author{
Frank J. Christophersen, Melanie N. Zeilinger, Colin N. Jones, and Manfred Morari \\ Automatic Control Laboratory, ETH Zurich, Physikstrasse 3, ETL K13.1, CH-8092 Zurich, Switzerland \\ fjc|zeilinger|cjones | morari@control.ee.ethz.ch
}

\begin{abstract}
We consider the class of piecewise affine optimal state feedback control laws applied to discrete-time piecewise affine systems, motivated by recent work on the computation of closed-form MPC controllers. The storage demand and complexity of these optimal closed-form solutions limit their applicability in most real-life situations. In this paper we present a novel algorithm to a posteriori reduce the storage demand and complexity of the closed-form controller without losing closed-loop stability or all time feasibility while guaranteeing a bounded performance decay compared to the optimal solution. The algorithm combines simple polyhedral manipulations with (multi-parametric) linear programming and the effectiveness of the algorithm is demonstrated on a large numerical example.
\end{abstract}

Keywords - constrained systems, discrete-time systems, piecewise affine systems, receding horizon control, MPC, closed-form solution, lookup table, multi-parametric programming, optimal control, complexity reduction, approximation.

\section{INTRODUCTION}

This work is motivated by the recent developments in the field of controller synthesis for hybrid systems [1], [2], [3], [4], [5], [6]. A significant amount of the research in this field has focused on solving constrained optimal control problems, both for continuous-time and discrete-time hybrid systems.

We consider the class of constrained discrete-time piecewise affine (PWA) systems [3] that are obtained by partitioning the extended state-input space into polyhedral regions and associating with each region a different affine state update equation. As shown in [7], the class of piecewise affine systems is of rather general nature and equivalent to many other hybrid system formalisms reported in the literature.

For piecewise affine systems the constrained finite time optimal control (CFTOC) problem can be solved by means of multi-parametric programming [4], [6], [8] and the resulting closed-form solution is a time-varying PWA state feedback control law. If the solution to the CFTOC problem is used in a receding horizon control ( $\mathrm{RHC}$ ) (or model predictive control (MPC)) [9], [10] strategy then the time-varying PWA state feedback control law becomes time-invariant and can serve as a control 'lookup table' on-line, thus enabling RHC to be used for fast sampled systems.

However, due to the combinatorial nature of the considered problem the number of state space regions over which the control lookup table is defined grows in the worst case exponentially [4], [11]. Therefore, firstly, efficient on-line search strategies [12], [6] are required to achieve fast sampling rates and/or, secondly, in order to cope with the limited storage and memory of most control devices, a reduction of the complexity (i.e. mainly the number of defining state space regions of the controller) or approximation of the optimal control lookup table is essential.

Several authors recently addressed the complexity reduction or approximation issue by either modifying the original CFTOC problem, retrieving a suboptimal solution of the CFTOC problem, or by post-processing the computed optimal controller, cf. e.g. [13], [14], [15]. However, a direct guarantee on the reduction of the complexity, closed-loop stability, or performance decay is mostly neglected.

In this paper we present a novel algorithm to a posteriori reduce the storage demand and complexity of the closedform control lookup table without losing closed-loop stability or all time feasibility while guaranteeing a bounded performance decay compared to the optimal solution. The algorithm combines simple polyhedral manipulations with (multi-parametric) linear programming and the effectiveness of the algorithm is demonstrated on a large numerical example.

\section{Constrained Finite Time Optimal Control of PIECEWISE AFFINE Systems}

Piecewise affine (PWA) systems are equivalent to many other hybrid system classes [3], [7] such as mixed logical dynamical systems [16], linear complementary systems [2], and max-min-plus-scaling systems [17] and thus form a very general class of linear hybrid systems.

We consider the class of discrete-time, stabilizable, linear hybrid systems that can be described as constrained PWA systems of the following form

$$
\begin{aligned}
x(t+1) & =f_{\mathrm{PWA}}(x(t), u(t)) \\
& :=A_{d} x(t)+B_{d} u(t)+a_{d}, \quad \text { if }\left[\begin{array}{l}
x(t) \\
u(t)
\end{array}\right] \in \mathcal{D}_{d},
\end{aligned}
$$

where $t \geq 0$, the domain $\mathcal{D}:=\cup_{d=1}^{N_{\mathcal{D}}} \mathcal{D}_{d}$ of $f_{\mathrm{PWA}}(\cdot, \cdot)$ is a non-empty compact set in $\mathbb{R}^{n_{x}+n_{u}}$ with $N_{\mathcal{D}}<\infty$ the number of system dynamics, and $\left\{\mathcal{D}_{d}\right\}_{d=1}^{N_{\mathcal{D}}}$ denotes a polyhedral partition of the domain $\mathcal{D}$, i.e. the closure of $\mathcal{D}_{d}$ is $\overline{\mathcal{D}}_{d}:=\left\{\left[\begin{array}{l}x \\ u\end{array}\right] \in \mathbb{R}^{n_{x}+n_{u}} \mid D_{d}^{x} x+D_{d}^{u} u \leq D_{d}^{0}\right\}$ and $\operatorname{int}\left(\mathcal{D}_{d}\right) \cap \operatorname{int}\left(\mathcal{D}_{j}\right)=\emptyset$ for all $d \neq j$. 
Remark II.1 (Constraints). Note that linear state constraints $\left(x(t) \in \mathbb{X} \subseteq \mathbb{R}^{n_{x}}\right)$ and input constraints $(u(t) \in \mathbb{U} \subseteq$ $\mathbb{R}^{n_{u}}$ ) of the general form $C^{x} x+C^{u} u \leq C^{0}$ are naturally incorporated in the description of $\mathcal{D}_{d}$.

The following is assumed throughout this work

Assumption II.2 (Equilibrium at the origin). The origin in the extended state-input space is an equilibrium point of the PWA system (1), i.e. $\mathbb{O}_{n_{x}+n_{u}} \in \mathcal{D}$ and $\mathbb{O}_{n_{x}}=$ $f_{\mathrm{PWA}}\left(\mathbb{O}_{n_{x}}, \mathbb{O}_{n_{u}}\right)$, where $\mathbb{O}_{n}:=\left[\begin{array}{llll}0 & 0 & \ldots & 0\end{array}\right]^{\prime} \in \mathbb{R}^{n}$.

The above assumption does not limit the scope of this work, i.e. the presented results also hold for any non-zero equilibrium point.

The flexibility of this modeling framework and the recent technological advances in the fields of optimization and control theory have lead to a considerable interest in academia and industry in PWA systems; not to mention that many engineering systems naturally express themselves or can be approximated nicely by PWA systems.

\section{A. Constrained Finite Time Optimal Control}

We define for the aforementioned PWA system (1) the constrained finite time optimal control (CFTOC) problem

$$
\begin{aligned}
& J_{T}^{*}(x(0)):=\min _{U_{T}} J_{T}\left(x(0), U_{T}\right) \\
& \text { s.t. }\left\{\begin{array}{l}
x(t+1)=f_{\mathrm{PWA}}(x(t), u(t)) \\
x(T) \in \mathcal{X}^{f},
\end{array}\right.
\end{aligned}
$$

where

$$
J_{T}\left(x(0), U_{T}\right):=\ell_{T}(x(T))+\sum_{t=0}^{T-1} \ell(x(t), u(t))
$$

is the cost function, $\ell(\cdot, \cdot)$ the stage cost,$\ell_{T}(\cdot)$ the final penalty function, $U_{T}$ is the optimization variable defined as the input sequence $U_{T}:=\{u(t)\}_{t=0}^{T-1}, T<\infty$ is the prediction horizon, and $\mathcal{X}^{f}$ is a compact terminal target set in $\mathbb{R}^{n_{x}}$. With a slight abuse of notation, when the CFTOC problem (2a)-(2b) has multiple solutions, i.e. when the optimizer is not unique, $U_{T}^{*}(x(0)):=\left\{u^{*}(t)\right\}_{t=0}^{T-1}$ denotes one (arbitrarily chosen) realization from the set of possible optimizers.

The CFTOC problem (2a)-(2b) implicitly defines the set of feasible initial states $\mathcal{X}_{T} \subset \mathbb{R}^{n_{x}}\left(x(0) \in \mathcal{X}_{T}\right)$ and the set of feasible inputs $\mathcal{U}_{T-t} \subset \mathbb{R}^{n_{u}}\left(u(t) \in \mathcal{U}_{T-t}, t=0, \ldots, T-\right.$ $1)$. In the context of this paper, the goal in this section is to give an explicit (closed-form) expression for $u^{*}(t): \mathcal{X}_{T} \rightarrow$ $\mathcal{U}_{T-t}, t=0, \ldots, T-1$.

Consider the two following restrictions to the CFTOC problem

Problem II.3 (PWA system, 1-/ $\infty$-norm based cost).

$$
\begin{aligned}
\ell(x(t), u(t)) & :=\|Q x(t)\|_{p}+\|R u(t)\|_{p}, \\
\ell_{T}(x(T)) & :=\|P x(T)\|_{p},
\end{aligned}
$$

where $\|\cdot\|_{p}$ with $p \in\{1, \infty\}$ denotes the standard vector $1-/ \infty$-norm [18], [6], and

Problem II.4 (Constr. LTI system, quadratic cost).

$$
\begin{aligned}
f_{\mathrm{PWA}}(x(t), u(t)) & :=A x(t)+B u(t), \text { if }\left[\begin{array}{l}
x(t) \\
u(t)
\end{array}\right] \in \mathcal{D}, \\
\ell(x(t), u(t)) & :=x(t)^{\prime} Q x(t)+u(t)^{\prime} R u(t), \\
\ell_{T}(x(T)) & :=x(T)^{\prime} P x(T) .
\end{aligned}
$$

In both CFTOC Problem II.3 and II.4 the solution is a time-varying PWA state feedback control law defined over a polyhedral partition, which is stated in the following theorem and proved in e.g. [19], [4].

Theorem II.5 (Solution to CFTOC). The solution to the optimal control problem (2a)-(2b), restricted to Problem II.3 or II.4, is a time-varying PWA function of the initial state $x(0)$

$$
\mu_{\mathrm{PWA}}(x(0), t)=K_{T-t, i} x(0)+L_{T-t, i}, \quad \text { if } \quad x(0) \in \mathcal{P}_{i}
$$

with $u^{*}(t)=\mu_{\mathrm{PWA}}(x(0), t)$, where $t=0, \ldots, T-1$, and $\left\{\mathcal{P}_{i}\right\}_{i=1}^{N_{\mathcal{P}}}$ is a polyhedral partition of the set of feasible states $x(0), \mathcal{X}_{T}=\cup_{i=1}^{N_{\mathcal{P}}} \mathcal{P}_{i}$, with the closure of $\mathcal{P}_{i}$ given by $\overline{\mathcal{P}}_{i}=$ $\left\{x \in \mathbb{R}^{n_{x}} \mid P_{i}^{x} x \leq P_{i}^{0}\right\}$.

In the case that a receding horizon $(\mathrm{RH})$ control policy or a model predictive controller (MPC) [9], [10] is used in closedloop, the control is given as a time-invariant state feedback control law of the form

$$
\mu_{\mathrm{RH}}(x(t)):=K_{T, i} x(t)+L_{T, i}, \quad \text { if } \quad x(t) \in \mathcal{P}_{i},
$$

where $i=1, \ldots, N_{\mathcal{P}}$ and $u(t)=\mu_{\mathrm{RH}}(x(t))$ for $t \geq 0$.

Definition II.6 (Feasibility for all time). A CFTOC problem is called feasible at time $t$ if there exists a control action at time $t$ for the measured state $x_{t}=: x(0)$, which satisfies the state and input constraints over the considered prediction horizon T. A RHC problem is called feasible for all time if it is feasible for all $t \geq 0$.

Assumption II.7 (Stability, feasibility). Note that in the following it is assumed that the parameters $T, Q, R, P$, and $\mathcal{X}^{f}$ are chosen in such a way that (5) is closed-loop stabilizing, feasible for all time and that a polyhedral PWA Lyapunov function [20], [21] of the form

$$
V(x)=V_{i}^{x} x+V_{i}^{0}, \quad \text { if } x \in \mathcal{P}_{i},
$$

where $i=1, \ldots, N_{\mathcal{P}}$, for the closed-loop system

$$
x(t+1)=f^{\mathrm{CL}}(x(t)):=f_{\mathrm{PWA}}\left(x(t), \mu_{\mathrm{RH}}(x(t))\right),
$$

$x(t) \in \mathcal{X}_{T}$, exists and is given.

This is usually not a restricting requirement but rather the aim of most (if not all) control strategies. Furthermore, we remark that if the parameters are chosen according to e.g. [9], [22], or [6] one can simply take $V(\cdot)=J_{T}^{*}(\cdot)$.

In the course of this paper our focus lies on the reduction of the complexity of the closed-form control law $\mu_{\mathrm{RH}}(\cdot)$ 
without losing closed-loop stability nor feasibility for all time.

\section{COMPLEXITY REDUCTION}

As the system dimension $n_{x}$ and control dimension $n_{u}$ are fixed, the storage demand (or complexity) of a closed-form control law (5) is influenced solely by the defining polyhedral partition, i.e. the number $N_{\mathcal{P}}$ of defining state space polyhedral regions $\mathcal{P}_{i}$ and the number of their respective facets [23]. Unfortunately, depending on the structure and parameters of the underlying system and optimization problem, one of the main drawbacks with optimal closed-form control laws $\mu(\cdot)$ is the possible worst case exponential 'explosion' [4], [11] in the number of regions $N_{\mathcal{P}}$. But even in an average case the number $N_{\mathcal{P}}$ tends to be very large and above the storage limit of most control devices. Therefore, it is often essential for a real-life implementation of the closed-form solution to find an appropriate approximation of the controller or a controller with reduced complexity.

As mentioned in the introduction, several authors recently addressed the issue of complexity reduction or approximation by either modifying the original CFTOC problem, retrieving a suboptimal solution of the CFTOC problem, or by postprocessing the computed optimal controller. The authors in [14], [24], for example, aim at computing a minimal polyhedral representation of the original controller partition in order to reduce storage complexity. However, the computation is 'practically' limited to a small number of regions with a small number of facets, since the computation time grows exponentially. A different proposal is given in [13], where the original CFTOC problem (for constrained linear systems) is modified a priori by relaxing the underlying multi-parametric program in order to find an approximate, hopefully simpler, solution.

However, most proposals in the literature lack a guarantee on the reduction of the complexity, closed-loop stability, or maximal performance decay.

The here proposed post-processing strategy aims at a direct reduction of the controller complexity by a 'safe' elimination (i.e. removal) of 'small' regions, whereby closedloop stability and all time feasibility is not lost and a bounded performance decay (compared to the optimal solution) can be guaranteed. The resulting controller partition will have 'holes' in the feasible state space $\mathcal{X}_{T}$. Then in the on-line control procedure (Section IV) a fast alternative strategy is implemented for the case that the measured state lies in a safely eliminated region. This leads to a direct reduction of the storage requirements and on-line computation of the approximate controller.

The algorithm and derivation is detailed in the following.

\section{A. Safe Region Elimination}

Simulations (and in the case of constrained linear system also continuity of the respective functions) seem to indicate that the impact of 'small' regions (i.e. regions with either a small Chebyshev radius [25], small volume, or small Lyapunov decay $\Delta V$ ) have in practice little influence on the closed-loop stability and performance of the overall system, when perturbing or approximating the control law inside of these regions. Other factors reducing the importance of small regions are for example: due to real measurement noise it is unlikely that the measured state lies in or enters such regions and, furthermore, these regions tend to often occur in areas of the state space away from a neighborhood around the equilibrium point and thus the time that the system trajectory 'spends' in these areas is minimal.

In order to present Algorithm III.3 for the safe elimination of regions, the two core ideas behind the algorithm need to be explained. The first idea is based on the inherent freedom of the Lyapunov decay inequality (7c) of Theorem III.1, repeated for completeness in the following and proved in [21].

Theorem III.1 (Asymptotic/exponential stability). Let $\mathcal{X}_{T}$ be a bounded positively invariant set in $\mathbb{R}^{n_{x}}$ for the autonomous (closed-loop) system (6) and let $\underline{\alpha}(\cdot), \bar{\alpha}(\cdot)$, and $\beta(\cdot)$ be $K$-class functions [20]. If there exists a non-negative function $V: \mathcal{X}_{T} \rightarrow \mathbb{R}_{\geq 0}$ with $V\left(\mathbb{O}_{n_{x}}\right)=0$ such that

$$
\begin{aligned}
V(x) & \geq \underline{\alpha}(\|x\|), \\
V(x) & \leq \bar{\alpha}(\|x\|), \\
\Delta V(x):=V\left(f^{\mathrm{CL}}(x)\right)-V(x) & \leq-\beta(\|x\|),
\end{aligned}
$$

where $x \in \mathcal{X}_{T}$, then the following results holds:

(a) The equilibrium point $\mathbb{O}_{n_{x}}$ is asymptotically stable [20] in the Lyapunov sense in $\mathcal{X}_{T}$.

(b) If $\underline{\alpha}(\|x\|):=\underline{a}\|x\|^{\gamma}, \bar{\alpha}(\|x\|):=\bar{a}\|x\|^{\gamma}$, and $\beta(\|x\|):=$ $b\|\bar{x}\|^{\gamma}$ for some positive constants $\underline{a}, \bar{a}, b, \gamma>0$ then the equilibrium point $\mathbb{O}_{n_{x}}$ is exponentially stable [20] in the Lyapunov sense in $\mathcal{X}_{T}$.

Simply speaking, if all the prerequisites of Theorem III.1 are fulfilled with a given controller $\mu_{\mathrm{RH}}(\cdot)$, the resulting behavior of the closed-loop system is stabilizing. If, for the given Lyapunov function $V(\cdot), \beta(\cdot)$ is now relaxed, one can (possibly) find a set of controllers that will render the closedloop system stabilizing and feasible. (Note, that setting $\beta(\bullet)$ close to the zero-function is sufficient for pure asymptotic stability.)

For the considered class of PWA systems, PWA control laws, and PWA Lyapunov functions with $\beta(\cdot)$ consisting of a sum of weighted vector $1-/ \infty$-norms, the Lyapunov decay inequality (7c) describes a collection of polytopic sets. This enables a stability test for altered PWA control laws with simple feasibility LPs. Thus the idea, originally motivated by continuity arguments, is to test if a control function $\widetilde{\mu}(\cdot)$ of a 'close by' state space area, evaluated at the local state $x$ still fulfills the Lyapunov decay inequality (7c) and thus stabilizes the overall closed-loop system. 


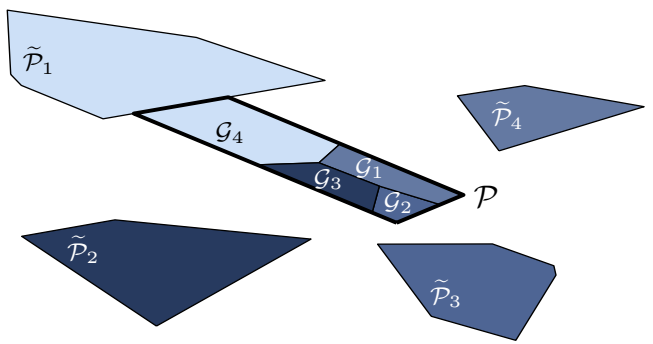

Fig. 1: Example of a minimal-violation partition $\left\{\mathcal{G}_{j}\right\}_{j=1}^{4}$ of $\mathcal{P}$ in $\mathbb{R}^{n_{x}}$ with respect to $\left\{\widetilde{\mathcal{P}}_{i}\right\}_{i=1}^{4}$. The corresponding index function $l(x)$ is indicated by the corresponding coloring of the regions.

This leads to the second idea which is based on a new distance concept called minimal-violation distance defined in the following.

Definition III.2 (Minimal-violation distance). Let the collection $\widetilde{\mathcal{P}}$ be the set $\widetilde{\mathcal{P}}:=\left\{\widetilde{\mathcal{P}}_{i}\right\}_{i=1}^{N_{\tilde{\mathcal{P}}}}$ where $\widetilde{\mathcal{P}}_{i}:=\{x \in$ $\left.\mathbb{R}^{n_{x}} \mid \widetilde{\mathcal{P}}_{i}^{x} x \leq \widetilde{\mathcal{P}}_{i}^{0}\right\}$ are full-dimensional polyhedra ${ }^{1}$ in $\mathbb{R}^{n_{x}}$. The minimal-violation distance $d_{\mathrm{MV}}$ of $x$ to $\widetilde{\mathcal{P}}$ is given by

$$
\begin{aligned}
d_{\mathrm{MV}}(x, \widetilde{\mathcal{P}}) & :=\min _{i}\left\{\alpha_{i}^{*}(x)\right\}_{i}, \quad \text { where } \\
\alpha_{i}^{*}(x) & =\min \left\{\alpha_{i} \in \mathbb{R} \mid \widetilde{\mathcal{P}}_{i}^{x} x \leq \widetilde{\mathcal{P}}_{i}^{0}+\alpha_{i} \mathbb{\mathbb { 1 }}\right\},
\end{aligned}
$$

for all $i=1, \ldots, N_{\widetilde{\mathcal{P}}}$ and $\mathbb{1}:=\left[\begin{array}{llll}1 & 1 & \ldots & 1\end{array}\right]^{\prime}$.

If $x \in \mathcal{P}$, where $\mathcal{P}$ is some polytope, one obtains that $d_{\mathrm{MV}}(\cdot, \widetilde{\mathcal{P}})$ is a (possibly non-convex) polyhedral PWA function of the form

$$
d_{\mathrm{MV}}(x, \widetilde{\mathcal{P}})=d_{j}^{x} x+d_{j}^{0}, \quad \text { if } \quad x \in \mathcal{G}_{j},
$$

where $\mathcal{G}:=\left\{\mathcal{G}_{j}\right\}_{j=1}^{N_{\mathcal{G}}}$ is a polyhedral partition of $\mathcal{P}$. (Note, that $N_{\mathcal{G}}$ can be large, however, the partition $\mathcal{G}$ is only used during the post-processing step and has no influence on the on-line controller evaluation, cf. Algorithm III.3 and IV.1.) The PWA function (9) can be obtained by, for example, first solving the single multi-parametric linear program [4]

$$
\begin{array}{ll}
\min & \sum_{i=1}^{N_{\tilde{\mathcal{P}}}} \alpha_{i} \\
\text { subj. to } & \widetilde{\mathcal{P}}_{i}^{x} x \leq \widetilde{\mathcal{P}}_{i}^{0}+\alpha_{i} \mathbb{1}, \forall i=1, \ldots, N_{\tilde{\mathcal{P}}}, \\
& x \in \mathcal{P} .
\end{array}
$$

Within each critical region [4] of (10), the functions $\alpha_{i}^{*}(x)$ are affine, rather than piecewise affine. It is therefore straightforward to compute $d_{\mathrm{MV}}(x, \widetilde{\mathcal{P}})=\min _{i}\left\{\alpha_{i}^{*}(x)\right\}_{i}$ within each critical region by determining the irredundant inequalities [23] of the convex polytope $\left\{(d, x) \mid d \leq \alpha_{i}^{*}(x), \forall i\right\}$.

The 'closest' index function

$$
l(x):=\left\{i \in\left\{1, \ldots, N_{\widetilde{\mathcal{P}}}\right\} \mid d_{\mathrm{MV}}(x, \widetilde{\mathcal{P}})=\alpha_{i}^{*}(x)\right\},
$$

$x \in \mathcal{P}$, is constant for all points $x \in \mathcal{G}_{j}$ and indicates the index of $\widetilde{\mathcal{P}}_{i}$ which is 'closest' (i.e. has the smallest violation)

\footnotetext{
${ }^{1}$ We assume that $\widetilde{P}_{i}^{x} x(t) \leq \widetilde{P}_{i}^{0}$ is in the Hessian normal form, i.e. each row $\left[\widetilde{P}_{i}^{x}\right]_{r}$ of $\widetilde{P}_{i}^{x}$ is normalized with $\left\|\left[\widetilde{P}_{i}^{x}\right]_{r}\right\|_{2}=1$.
}

to $x$. In the following $\mathcal{G}$ will be called the minimal-violation partition of $\mathcal{P}$ with respect to $\left\{\widetilde{\mathcal{P}}_{i}\right\}_{i=1}^{N_{\tilde{\mathcal{P}}}}$. Refer to Figure 1 for an example.

This measure enables the detection of the 'closest' neighboring regions and respective controllers in the on-line controller evaluation without the need to additionally solve on-line optimization problems, cf. Section IV.

This leads to the following overall post-processing Algorithm III.3:

\section{Algorithm III.3 (Safe region elimination)}

$$
\begin{aligned}
& \text { INPUT } \quad f_{\mathrm{PWA}}(x, u), \mu_{\mathrm{RH}}(x), V(x), \mathcal{X}_{T},\left\{\mathcal{P}_{i}\right\}_{i=1}^{N_{\mathcal{P}}} \\
& \text { OUTPUT } \widetilde{\mu}_{\mathrm{RH}}(x),\left\{\mathcal{P}_{i}\right\}_{\tilde{\mathcal{I}}} \\
& \mathcal{I}_{\text {remove }} \leftarrow \emptyset, \mathcal{I}_{\text {keep }} \leftarrow\left\{1, \ldots, N_{\mathcal{P}}\right\} \\
& \text { FOR EACH } i_{\text {remove }} \in\left\{1, \ldots, N_{\mathcal{P}}\right\} \\
& \quad \text { FOR EACH } j \in \mathcal{I}_{\text {remove }} \cup i_{\text {remove }}
\end{aligned}
$$

(a) Compute the minimal-violation partition $\left\{\mathcal{G}_{k}^{j}\right\}_{k=1}^{N_{\mathcal{G}^{j}}}$ and corresponding closest index set $\left\{l_{k}^{j}\right\}_{k=1}^{N_{\mathcal{G}^{j}}}$ for region $j$ with respect to $\mathcal{I}_{\text {keep }} \backslash i_{\text {remove }}$

(b) Check for all $k=1, \ldots, N_{\mathcal{G}^{j}}$ if the control law of the closest region $l_{k}^{j}$ is valid for all $x \in \mathcal{G}_{k}^{j}$, i.e.

$$
\begin{gathered}
\mu_{l_{k}^{j}}(x):=K_{T, l_{k}^{j}} x+L_{T, l_{k}^{j}} \in \mathbb{U}, \\
f_{\mathrm{PWA}}\left(x, \mu_{l_{k}^{j}}(x)\right) \in \mathcal{X}_{T}, \\
V\left(f_{\mathrm{PWA}}\left(x, \mu_{l_{k}^{j}}(x)\right)\right)-V(x) \leq-\beta(\|x\|)
\end{gathered}
$$

IF all in (b) are valid THEN

$$
\begin{aligned}
\mathcal{I}_{\text {keep }} & \leftarrow \mathcal{I}_{\text {keep }} \backslash i_{\text {remove }} \\
\mathcal{I}_{\text {remove }} & \leftarrow \mathcal{I}_{\text {remove }} \cup i_{\text {remove }} \\
\text { END } & \\
\text { END } &
\end{aligned}
$$

END

$\widetilde{\mu}_{\mathrm{RH}}(x):=K_{T, i} x+L_{T, i}, \quad$ if $\quad x \in \mathcal{P}_{i}, \quad$ and $\quad i \in \mathcal{I}_{\text {keep }}$

Note that the test in (11) can be solved by simple feasibility LPs. Moreover, the minimal-violation partition in step (a) only needs to be recomputed if the region to be removed is contained in the index set $\left\{l_{k}^{j-1}\right\}_{k=1}^{N_{\mathcal{G}^{j-1}}}$. Additionally, various heuristics to speed up and enhance the algorithm can be applied such as, for example, the consideration of only the regions in the outer FOR-loop which are likely to pass the test in (b) (i.e. for example regions with a small Chebyshev radius, a small volume, or a large number of facets) or to modify the strict iterative behavior of the algorithm by applying and testing the inner FOR-loop on batches of regions at once.

Furthermore, we point out that the algorithm operates in a greedy fashion and therefore does not necessarily remove the maximal number of regions. An optional procedure is certainly possible by the addition of a backtracking procedure in order to find the optimal removal order, although this is unlikely to be computationally feasible in practice. 


\section{B. Additional Performance Constraint}

As mentioned before, if the Lyapunov decay rate $\beta(\cdot)$ in inequality (11c) of Algorithm III.3 is chosen to be close to the zero-function, i.e. for example $V\left(f^{\mathrm{CL}}(x)\right)-V(x) \leq$ $-\beta\|x\|_{\infty}$ with $0<\beta \ll 1$, one guarantees an asymptotically stabilizing closed-loop system.

However, with the choice of $\beta(\cdot)$ a detuning of the closedloop performance $\sum_{t=0}^{\infty} \ell(x(t), u(t))$, with some control law $u(t)=\widetilde{\mu}(x(t))$, compared to the optimal RHC solution $\mu_{\mathrm{RH}}(\cdot)$ can be performed. Thus one can, for example, try to remove 'small' regions without losing closed-loop stability, all time feasibility, while still guaranteeing a given, bounded performance decay of $\eta \%$. How $\beta(\cdot)$ in step 3(c) of Algorithm III.3 needs to be modified is elaborated in the following.

Theorem III.4 (Performance bound, [6]). Let $V(\bullet)$ be a Lyapunov function for the closed-loop system $x(t+1)=$ $f(x(t), \mu(x(t))), \quad x \in \mathcal{X}$, under the stabilizing control $u(t)=\mu(x(t)) \in \mathbb{U}$ and let the prerequisites of Theorem III. 1 be fulfilled. Furthermore, let $\ell(x, u)$ be a $K$-class function determining the stage cost. Then every control law $u(t)=\widetilde{\mu}(x(t))$ with $x(t) \in \mathcal{X}$ (also any sequence of control samples $u(t))$ fulfilling

$$
\begin{gathered}
\widetilde{\mu}(x) \in \mathbb{U}, \\
f(x, \widetilde{\mu}(x)) \in \mathcal{X}, \\
V(f(x, \widetilde{\mu}(x)))-V(x) \leq-\beta \ell(x, \widetilde{\mu}(x)),
\end{gathered}
$$

for all $x \in \mathcal{X}$ with $\beta>0$, stabilizes the closed-loop system and guarantees a level of closed-loop performance given by

$$
\sum_{t=0}^{\infty} \ell(x(t), \widetilde{\mu}(x(t))) \leq \frac{1}{\beta} V(x(0)) .
$$

Proof. See [6, Ch. 10].

We remark, that from (13) it follows that the performance decay $\eta$ [in \%] with respect to $V(x(0))$ is related to $\beta>0$ via $\beta(\eta)=\left(1+\frac{\eta[\text { in } \%]}{100}\right)^{-1}$.

In the case that the constrained infinite time optimal control (CITOC) problem [6] for PWA systems is solved, i.e. (roughly speaking) considering the CFTOC problem (2) with $T \rightarrow \infty$, one obtains the optimal solution $\mu_{\infty}^{*}(\cdot)$ with corresponding value function $J_{\infty}^{*}(\bullet)$. It was proved in [6] that $J_{\infty}^{*}(\cdot)$ is a Lyapunov function for the closed-loop system.

Corollary III.5 (Performance bound). Assume $V(\cdot)=$ $J_{\infty}^{*}(\cdot)$. Then every control law $u(t)=\widetilde{\mu}(x(t))$ with $x(t) \in$ $\mathcal{X}$ (also any sequence of control samples $u(t)$ ) fulfilling the conditions of Theorem III.4 guarantees

$$
J_{\infty}^{*}(x(0)) \leq \sum_{t=0}^{\infty} \ell(x(t), \widetilde{\mu}(x(t))) \leq \frac{1}{\beta} J_{\infty}^{*}(x(0)),
$$

and $0<\beta \leq 1$.

Proof. See [6, Ch. 10].

\section{ON-Line Control Evaluation}

As mentioned above, in an on-line application the control action $u(t)=\widetilde{\mu}_{\mathrm{RH}}(x(t)) \in \mathbb{R}^{n_{u}}$ is defined by

$$
\widetilde{\mu}_{\mathrm{RH}}(x(t)):=K_{T, i} x(t)+L_{T, i}, \quad \text { if } \quad x(t) \in \mathcal{P}_{i},
$$

where $i \in \mathcal{I}_{\text {keep }}$.

In order to evaluate the control action $u(t)$ one needs to identify the state space region $\mathcal{P}_{i}$, at the sampling instance $t$, in which the measured state $x(t)$ lies. In the case that $x(t)$ is in one of the 'small' state space regions $\mathcal{P}_{j}, j \in \mathcal{I}_{\text {removed }}$, that were safely removed during the post-processing step (Algorithm III.3), the control law of the 'closest' neighboring non-removed region is evaluated at $x(t)$, as outlined in the following algorithm.

\section{Algorithm IV.1 (Control evaluation)}

1. Measure the state $x(t)$ at time instance $t$

2. IF $x(t) \in \mathcal{X}_{T}$ THEN

$$
\begin{aligned}
& i^{\star}=\underset{i}{\arg \min }\left\{\alpha_{i}\right\}_{i \in \tilde{\mathcal{I}}}, \quad \text { where } \\
& \quad \alpha_{i}:=\max P_{i}^{x} x(t)-P_{i}^{0}, \quad \forall i \in \mathcal{I}_{\text {keep }} \\
& \text { IF }\left|i^{\star}\right| \geq 1 \text { THEN pick one element } i^{\star} \\
& \text { ELSE } \\
& \text { problem is infeasible STOP } \\
& \text { END }
\end{aligned}
$$$$
\text { 3. Apply } u(t)=K_{T, i^{\star}} x(t)+L_{T, i^{\star}} \text { to the system }
$$

Note that Definition (15) of 'closest' neighboring region is in accordance to Definition III.2 and allows a very simple on-line search operation where an additional on-line optimization is not required. Moreover, if $x(t) \in \mathcal{P}_{i^{\star}}$ for some $i^{\star} \in \mathcal{I}_{\text {keep }}$ then the constraint violation $\alpha_{i^{\star}}$ will be nonpositive and thus the iteration in (15b) can be prematurely interrupted, while guaranteeing that region $i^{\star}$ will be chosen correctly.

\section{NumERICAL EXAMPLE}

Consider the 'Car on a PWA Hill' example described in detail in [6, Part II], in which a frictionless car with mass $m=1$ is moving horizontally on a piecewise affine 'environment', cf. Figure 2. The goal of the car is to climb to the top of a steep hill and then to maintain its position at the top (the origin), without falling from the piecewise affine environment.

The discrete-time model is given by the following constrained and discontinuous PWA system

$$
x(t+1)=\left[\begin{array}{cc}
1 & 1 / 2 \\
0 & 1
\end{array}\right] x(t)+\left[\begin{array}{l}
1 / 8 \\
1 / 2
\end{array}\right] u(t)+a(x(t)),
$$

where

$$
a(x(t))= \begin{cases}\mathbb{O}_{2}, & \text { if } \quad\left[\begin{array}{ll}
1 & 0
\end{array}\right] x(t) \in(1), \\
-\frac{1}{4} g \sin \left(20 \frac{\pi}{180}\right)\left[\begin{array}{l}
1 \\
2
\end{array}\right], & \text { if }\left[\begin{array}{ll}
1 & 0
\end{array}\right] x(t) \in(2), \\
\mathbb{O}_{2}, & \text { if }\left[\begin{array}{ll}
1 & 0
\end{array}\right] x(t) \in(3), \\
-\frac{1}{4} g \sin \left(-5 \frac{\pi}{180}\right)\left[\begin{array}{l}
1 \\
2
\end{array}\right], & \text { if }\left[\begin{array}{ll}
1 & 0
\end{array}\right] x(t) \in(4),\end{cases}
$$




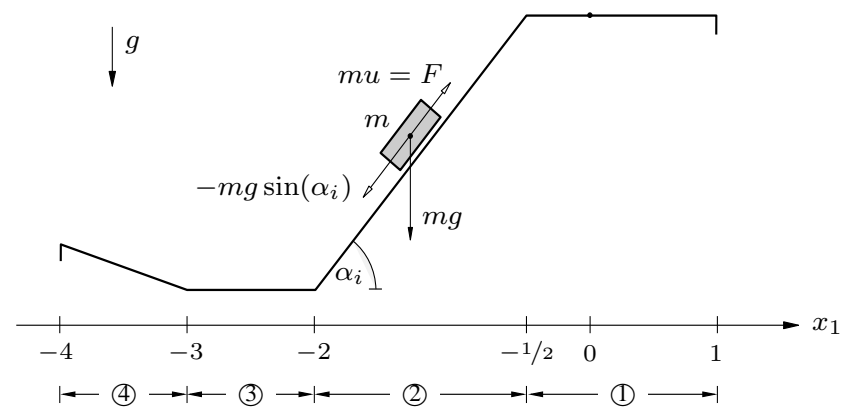

Fig. 2: Frictionless car moving on a piecewise affine hill.

$g$ is the gravitational constant, the first coordinate of $x$, i.e. $x_{1}$, is the horizontal position of the car, and the second, $x_{2}$, is its horizontal velocity. Moreover, the control action (i.e. force $F=m u$ acting on the car) is constrained by $|u(t)| \leq 2$ and $|u(t+1)-u(t)| \leq 40$ which prohibits the car to directly climbing up the steep hill in $x_{1} \in\left[-2,-\frac{1}{2}\right]$.

The CFTOC Problem II.3 was solved for $p=1, T=9$, $Q=\operatorname{diag}\left([100,1]^{\prime}\right)$, and $R=5 .\|P x\|_{1}$ with $P \in \mathbb{R}^{2 \times 2}$ and $\mathcal{X}^{f}$ were obtained with the algorithm described in [6, Ch. 8] in order to guarantee closed-loop stability. The optimal closed-form RHC solution $u(t)=\mu_{\mathrm{RH}}(x(t))$ was computed using the Multi-Parametric Toolbox (MPT) [26] for MATLAB ${ }^{\circledR}$ and comprises $N_{\mathcal{P}}=2083$ polyhedral state space regions.

The distribution of the Chebyshev radius, i.e. the 'smallness', of the regions of the optimal solution is depicted in the histogram in Figure 3. Note that more than $91 \%$ of all regions in the partition have a Chebyshev radius of 0.05 or smaller.

Using the proposed Algorithm III.3, it was possible to remove 1061 of 2083 regions safely, which constitutes a significant reduction of $50 \%$. While further simplification is possible, the computation was terminated after 16 hours, which is roughly twice the time required to compute the optimal controller. Note that no significant effort was invested in implementing the algorithm in an efficient manner and as such it is expected that a large reduction in the computation time is possible through the use of various heuristics and the combination with other post-processing simplification methods available in the literature.

\section{ACKNOWLEDGMENT}

We wish to thank Michal Kvasnica for his constructive and helpful comments.

\section{REFERENCES}

[1] A. van der Schaft and H. Schumacher, An Introduction to Hybrid Dynamical Systems, ser. Lecture Notes in Control and Information Sciences, M. Thoma, Ed. Springer-Verlag, 2000, vol. 251.

[2] W. P. M. H. Heemels, "Linear Complementarity Systems: A Study in Hybrid Dynamics," Ph.D. dissertation, Technische Universiteit Eindhoven, Eindhoven, The Netherlands, Nov. 1999.

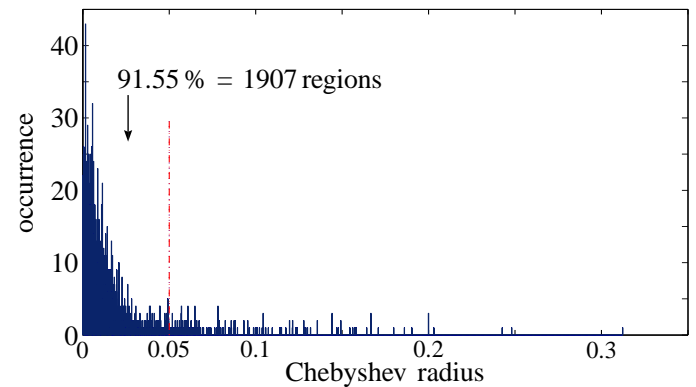

Fig. 3: Histogram for the Chebychev radius of the state space regions $\mathcal{P}_{i}$ of the solution to the example in Section V.

[3] E. D. Sontag, "Nonlinear regulation: The piecewise linear approach," IEEE Trans. on Automatic Control, vol. 26, no. 2, pp. 346-358, Apr. 1981.

[4] F. Borrelli, Constrained Optimal Control of Linear and Hybrid Systems, ser. Lecture Notes in Control and Information Sciences. Springer-Verlag, 2003, vol. 290.

[5] M. Johansson, Piecewise Linear Control Systems: A Computational Approach, ser. Lecture Notes in Control and Information Sciences, M. Thoma and M. Morari, Eds. Springer-Verlag, 2003, vol. 284.

[6] F. J. Christophersen, Optimal Control of Constrained Piecewise Affine Systems, ser. Lecture Notes in Control and Information Sciences. Springer Verlag, 2007, vol. 359.

[7] W. P. M. H. Heemels, B. De Schutter, and A. Bemporad, "Equivalence of hybrid dynamical models," Automatica, vol. 37, no. 7, pp. 10851091, 2001.

[8] E. C. Kerrigan and D. Q. Mayne, "Optimal control of constrained, piecewise affine systems with bounded disturbances," in Proc. of the Conf. on Decision \& Control, Las Vegas, Nevada, USA, Dec. 2002, pp. $1552-1557$.

[9] D. Q. Mayne, J. B. Rawlings, C. V. Rao, and P. O. M. Scokaert, "Constrained model predictive control: Stability and optimality," $\mathrm{Au}$ tomatica, vol. 36, no. 6, pp. 789-814, June 2000.

[10] J. M. Maciejowski, Predictive Control with Constraints. Prentice Hall, 2002.

[11] A. Bemporad, M. Morari, V. Dua, and E. N. Pistikopoulos, "The explicit linear quadratic regulator for constrained systems," Automatica, vol. 38, no. 1, pp. 3-20, Jan. 2002.

[12] P. Tøndel, T. A. Johansen, and A. Bemporad, "Evaluation of Piecewise Affine Control via Binary Search Tree," Automatica, vol. 39, no. 5, pp. 945-950, May 2003.

[13] A. Bemporad and C. Filippi, "Suboptimal explicit RHC via approximate multiparametric quadratic programming," Journal of Optimization Theory and Applications, vol. 117, no. 1, pp. 9-38, Apr. 2003.

[14] T. Geyer, F. D. Torrisi, and M. Morari, "Optimal Complexity Reduction of Piecewise Affine Models Based on Hyperplane Arrangements," in Proc. on the American Control Conference, Boston, Massachusetts, USA, June 2004, pp. 1190-1195.

[15] P. Grieder, "Efficient Computation of Feedback Controllers for Constrained Systems," Ph.D. dissertation, ETH Zurich, Automatic Control Laboratory, 2004.

[16] A. Bemporad and M. Morari, "Control of systems integrating logic, dynamics, and constraints," Automatica, vol. 35, no. 3, pp. 407-427, Mar. 1999.

[17] B. De Schutter and T. Van den Boom, "On model predictive control for max-min-plus-scaling discrete event systems," Automatica, vol. 37, no. 7, pp. 1049-1056, 2001.

[18] R. A. Horn and C. R. Johnson, Matrix Analysis. Cambridge University Press, 1985.

[19] D. Q. Mayne, "Constrained Optimal Control," European Control Conference, Plenary Lecture, Sept. 2001.

[20] M. Vidyasagar, Nonlinear Systems Analysis, 2nd ed. Prentice Hall, 1993.

[21] M. Lazar, "Model Predictive Control of Hybrid Systems: Stability and Robustness," Ph.D. dissertation, Technical University of Eindhoven, Eindhoven, The Netherlands, Sept. 2006.

[22] M. Lazar, W. P. M. H. Heemels, S. Weiland, and A. Bemporad, "Stabilizing Model Predictive Control of Hybrid Systems," IEEE Trans. on Automatic Control, vol. 51, no. 11, pp. 1813-1818, 2006. 
[23] B. Grünbaum, Convex Polytopes, 2nd ed., S. Axler, F. Gehring, and K. Ribet, Eds. Springer-Verlag, 2000.

[24] T. Geyer, F. D. Torrisi, and M. Morari, "Efficient Mode Enumeration of Compositional Hybrid Models," in Proc. of the Intern. Workshop on Hybrid Systems: Computation and Control, ser. Lecture Notes in Computer Science, vol. 2623. Springer-Verlag, 2003, pp. 216-232.

[25] S. Boyd and L. Vandenberghe, Convex Optimization. Cambridge University Press, 2004, http://www.stanford.edu/class/ee364/.

[26] M. Kvasnica, P. Grieder, and M. Baotić, "Multi-Parametric Toolbox (MPT)," 2004, available from http://control.ee.ethz.ch/ mpt/. 